\title{
Development of Sinusoidal BLDC Drive with Hall Sensors
}

\author{
Joon Sung Park ${ }^{\mathrm{a}, *}$, Jin-Hong Kimª ${ }^{\mathrm{a}}$, Byong Jo Hyon ${ }^{\mathrm{a}}$, Jun-Hyuk Choi ${ }^{\mathrm{a}}$, In-Soung Jung ${ }^{\mathrm{a}}$ \\ ${ }^{a}$ Korea Elctronics Technology Institute, \\ 203-101 Bucheon-TP B/D, Yakdae-dong, Wonmi-gu, Bucheon-si, Gyeonggi-do, 420-140, Korea \\ *Corresponding Author: parkjs@keti.re.kr
}

\begin{abstract}
In recent years, brushless DC (BLDC) motors are attracting growing attention for appliance applications. Due to the rapid development of technology, the BLDC motors are widely used in consumer electronics. Square wave method is used for most of small BLDC motor applications such as refrigerator fans, massage chairs, fans, compressors, etc. This is due to simple control and characteristics of the square wave method. However the square wave method has audible noise problem. In order to reduce this audible noise, the sinusoidal method is used. The sinusoidal method has a number of advantages such as high efficiency, low torque ripple, and low audible noise. The motor for appliance applications should be made increasing efficiency and decreasing the audible noise. However the sinusoidal method is complex and needs high cost. This paper deals primarily with the simple sinusoidal method for small BLDC motors.
\end{abstract}

Keywords: brushless DC motor, trapezoidal commutation, inverter, audible noise.

\section{Introduction}

Over the several years, DC motors have ever been prominent in various industrial applications because their characteristics and controls are simple. However DC motor systems have bulky construction, low efficiency, low reliability and need of maintenance [1]. Those features are unsuitable for appliance applications. In recent years, the BLDC motors are attracting growing attention for appliance applications. This is due to the total elimination of the brush/commutator assembly, which reduces audible noise and RFI problems. Moreover, BLDC motors have a number of advantages such as high efficiency, high power factor, and low maintenance cost. The motor for automotive applications should be made compact and increasing the efficiency causing limited volume. The operation of BLDC motors can be largely classified into two types which are trapezoidal commutation and sinusoidal commutation. The trapezoidal commutation method is used mainly in small BLDC because of the simple implementation. However the square wave method has audible noise problem. In order to reduce this audible noise, the sinusoidal method is used. The sinusoidal method has a number of advantages such as high efficiency, low torque ripple, and low audible noise. The motor for appliance applications should be made increasing efficiency and decreasing the audible noise. Based on the technological growth of power electronics, the trend in the appliance applications is to replace trapezoidal commutation method with sinusoidal commutation method based on electric motor technology [1]-[3]. In this paper, the sinusoidal method was applied to BLDC motors for appliance applications and decreased audible noise.

\section{Space Vector PWM}

The pulse-width modulation (PWM) is a DC voltage modulation method that provides an arbitrary intermediate voltage by adjusting the on and off ratio in each period. Numerous PWM methods were investigated by many researchers [4], [5], [6], but sinusoidal PWM and space vector PWM are the two most popular methods [3]. The sinusoidal PWM can be obtained by comparing a triangular wave with a sinusoidal wave. The maximum peak voltage is equal to $V_{d c} / 2$. However the maximum peak voltage of SVPWM is equal to $V_{d c} / \sqrt{3}$. Thus, the voltage utilization of SVPWM is better than SPWM.

The space vector PWM method [7],[8] (SVPWM) operates in a complex plane divided in the six sectors separated by the switching state vectors, Fig. 2. The switching state vectors are defined by combination of 
conducting/non-conducting switches in the power circuit of inverter. Fig. 1. The complex reference vector $V^{*}$ is used to locate two adjacent switching state vectors and to compute time $\left(T_{1}\right.$ and $\left.T_{2}\right)$ for which they are active. For the rest of sampling time $T_{s}$ zero switching state vectors $V_{7}$ or $V_{0}$ are active. SVPWM locally averages, over sampling period $T_{s}$, active and zero state vectors to be equal to the reference vector.

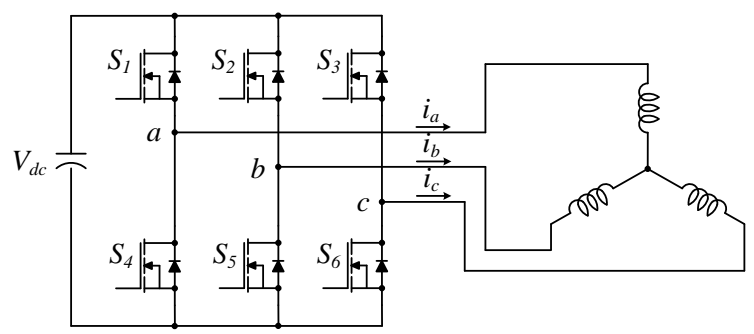

Fig. 1. Three phase inverter.

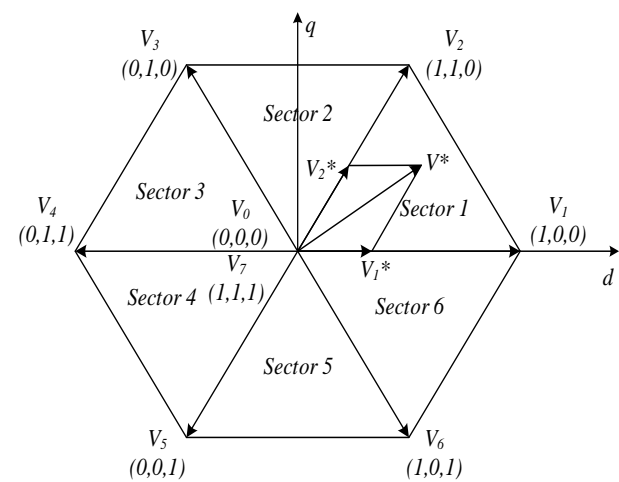

Fig. 2. Space vector diagram.

An arbitrary space vector can be represented as a sum of the decomposed vectors. Decomposed vector, $V_{l}{ }^{*}$, is synthesized by turning on $V_{I}(1,0,0)$ during a fractional time interval, $T_{l}$, which is proportional to the magnitude of $V_{l}{ }^{*}$. $T_{1}$ and $T_{2}$ can be determined as

$$
\begin{gathered}
\frac{V_{1}^{*}}{(2 / 3) V_{d c}}=\frac{T_{1}}{T_{s}}, \\
\frac{V_{2}^{*}}{(2 / 3) V_{d c}}=\frac{T_{2}}{T_{s}} .
\end{gathered}
$$

On the other hand, it follows from the geometrical relationship that

$$
\begin{aligned}
{\left[\begin{array}{c}
v_{d}^{*} \\
v_{q}^{*}
\end{array}\right] } & =V^{*}\left[\begin{array}{c}
\cos \theta \\
\sin \theta
\end{array}\right]=\frac{T_{1}}{T_{s}} \frac{2}{3} V_{d c}\left[\begin{array}{l}
1 \\
0
\end{array}\right]+\frac{T_{2}}{T_{s}} \frac{2}{3} V_{d c}\left[\begin{array}{c}
\cos (\pi / 3) \\
\sin (\pi / 3)
\end{array}\right] \\
& =\frac{2}{3} \frac{V_{d c}}{T_{s}}\left[\begin{array}{ll}
1 & \cos (\pi / 3) \\
0 & \sin (\pi / 3)
\end{array}\right]\left[\begin{array}{l}
T_{1} \\
T_{2}
\end{array}\right] .
\end{aligned}
$$

Therefore,

$$
\left[\begin{array}{l}
T_{1} \\
T_{2}
\end{array}\right]=\frac{T_{s} \sqrt{3}}{V_{d c}}\left[\begin{array}{cc}
\sin (\pi / 3) & -\cos (\pi / 3) \\
0 & 1
\end{array}\right]\left[\begin{array}{c}
v_{d}^{*} \\
v_{q}^{*}
\end{array}\right] .
$$

In all the six sectors, we can obtain time intervals $T_{1}$ and $T_{2}$.

$$
\begin{aligned}
& T_{1}=\frac{T_{s} \sqrt{3}}{V_{d c}}\left[\sin \left(\frac{\pi}{3} m\right) v_{d}^{*}-\cos \left(\frac{\pi}{3} m\right) v_{q}^{*}\right] \\
& T_{2}=\frac{T_{s} \sqrt{3}}{V_{d c}}\left[-\sin \left(\frac{\pi}{3}(m-1)\right) v_{d}^{*}+\cos \left(\frac{\pi}{3}(m+1)\right) v_{q}^{*}\right]
\end{aligned}
$$

From equation (4), (5), and (6), the time intervals T1, T2, and $\mathrm{T} 0$ can be obtained in the sector 1 .

$$
\begin{aligned}
& T_{1}=T_{s} \frac{\left|V^{*}\right|}{2 V_{d c} / 3} \frac{\sin \left(60^{\circ}-\theta\right)}{\sin 60^{\circ}} \\
& T_{2}=T_{s} \frac{\left|V^{*}\right|}{2 V_{d c} / 3} \frac{\sin (\theta)}{\sin 60^{\circ}} \\
& T_{0}=T_{s}-\left(T_{1}+T_{2}\right)
\end{aligned}
$$

By using above equations, the PWM can be decided. Fig. 3 shows the phase voltage represented by $\mathrm{S} 1, \mathrm{~S} 2$, and S3. These each phase voltages is not sinusoidal wave. Each phase voltage contains harmonics, so that the rms value is higher than that of the sinusoidal wave.

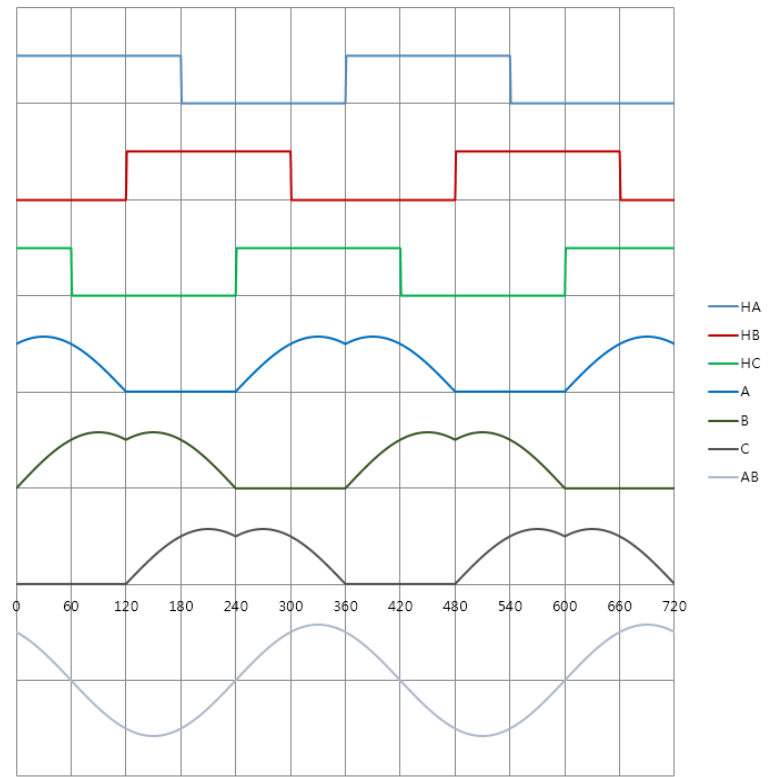

Fig. 3. Waveforms of hall signals, pole voltage, and line to line voltage 
SVPWM is complex and needs high speed processor. However the processing speed of the micro-processor for appliance applications is slow. The PWM generator was configured with PWM table. The sector can be distinguished by the hall sensors of BLDC motor. Therefore the PWM table is required for 60 degrees of interval, as shown in Fig. 4.

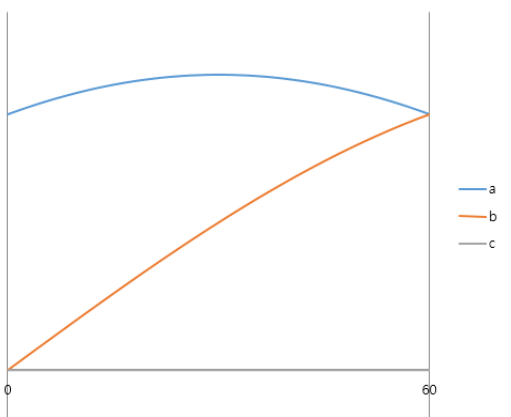

Fig. 4. Space vector output for $60^{\circ}$.

\section{Experimental Results}

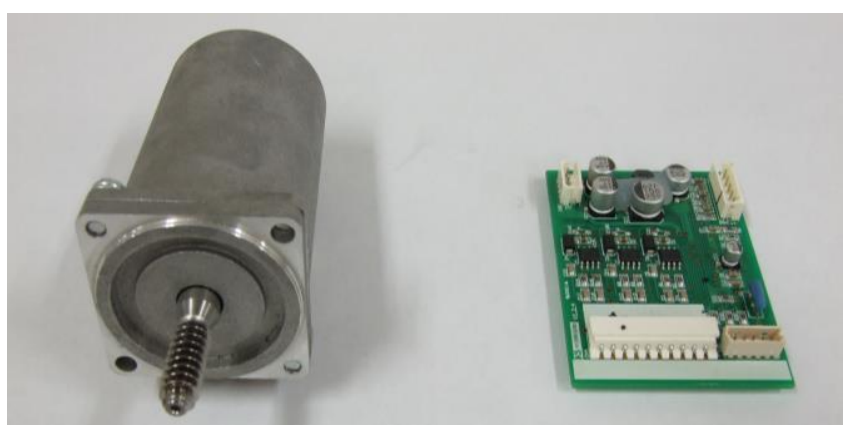

Fig. 5. Photos of BLDC motor and drive.

The BLDC motor and drive were tested extensuvly with dynamometer load and evaluated its performance over various test. Fig. 5 shows the BLDC motor and inverter. The switching frequency was selected $20 \mathrm{kHz}$ as shown in Fig. 6.

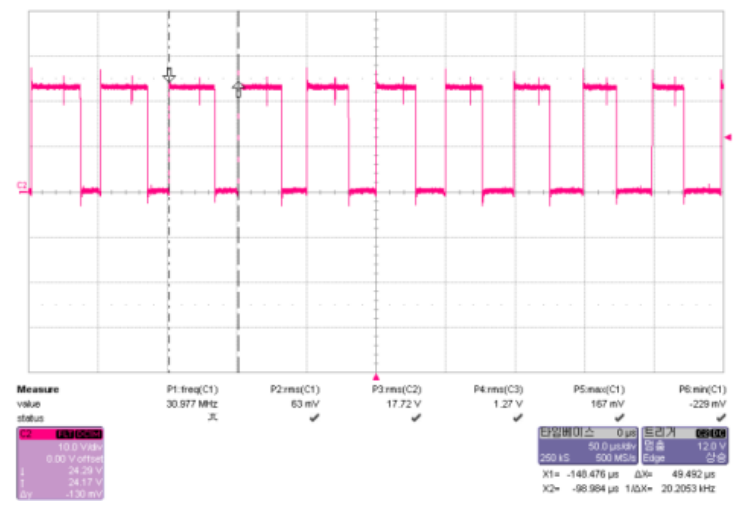

Fig. 6. PWM waveform.

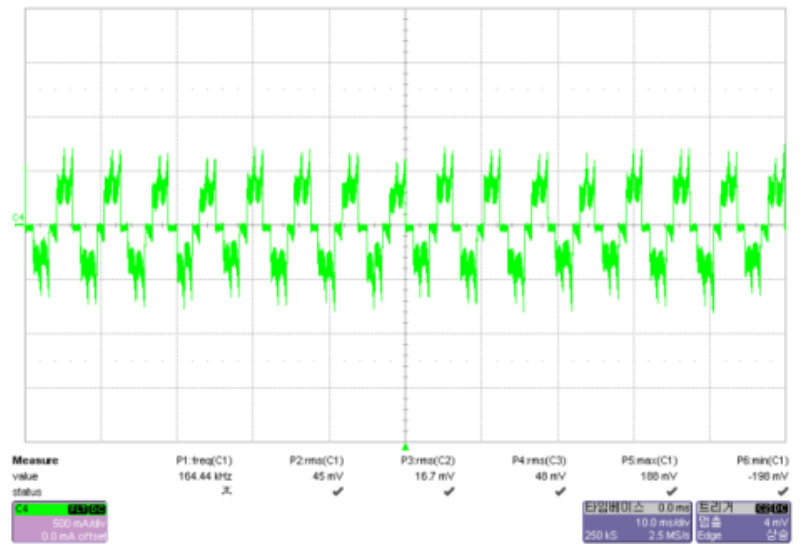

(a) Trapezoidal waveform

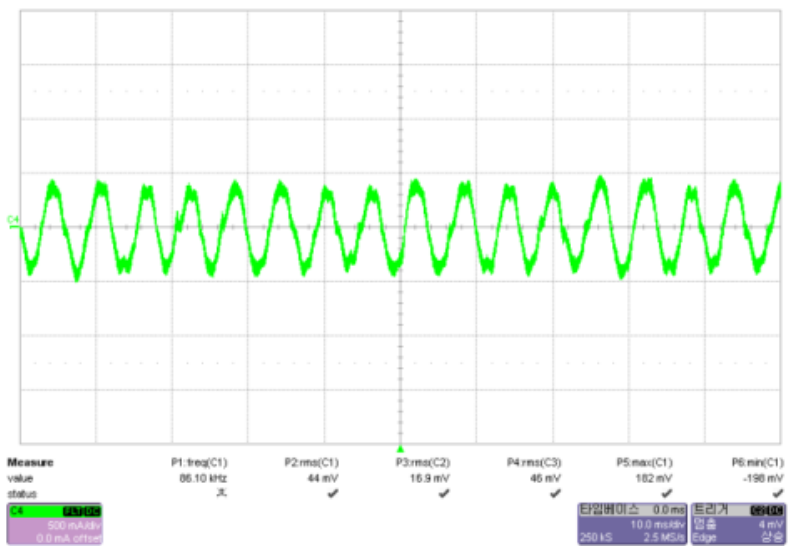

(b) Sinusoidal waveform

Fig. 7. Phase current.

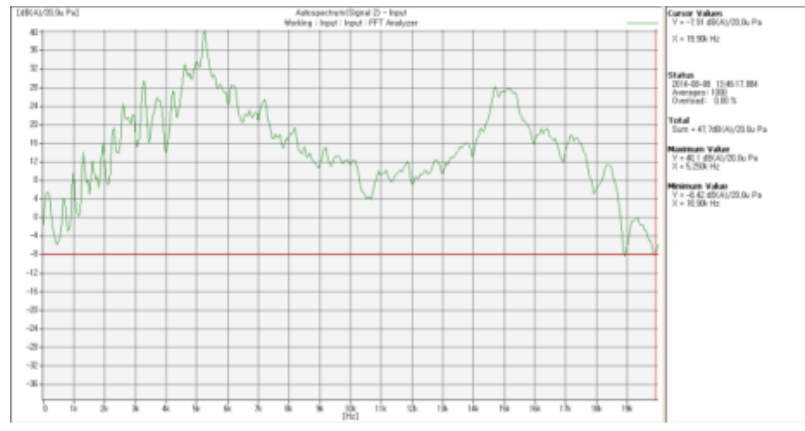

(a) Trapezoidal operation

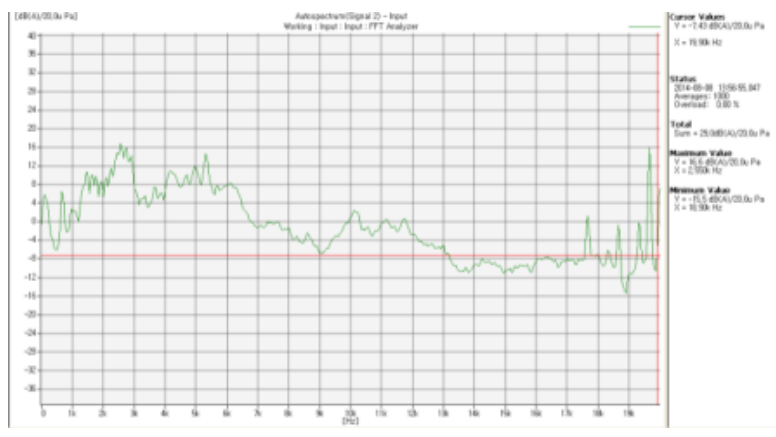

(b) Sinusoidal operation

Fig. 8. Noise test results. 


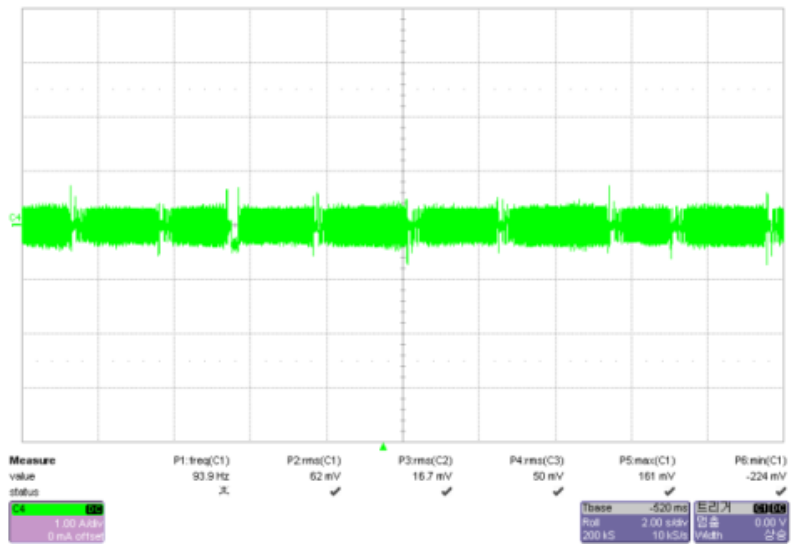

Fig. 9. Forward and reverse operation.

Fig. 7 shows the experimental waveforms in case of trapezoidal operation and sinusoidal operation. The peak current of sinusoidal operation is smaller than trapezoidal operation. To measure the noise level, the prototype motor and drive were tested in a dead room. Fig. 7 shows noise test results. The measured level of sinusoidal operation was $31.4 \mathrm{~dB}$. However the noise level of trapezoidal operation was $47.7 \mathrm{~dB}$. Fig. 9 shows the result of forward and reverse operation. The forward and reverse operation is operated well.

\section{Conclusion}

This paper presents a development of sinusoidal drive for appliance applications. Through the experimental results, validity and quality of the reported designs are verified, and developed motor is obtained the noise level $31.4 \mathrm{~dB}$ at the point of rated torque. Especially the excellent performance obtained. Also the drive has been developed by the method of space vector PWM, and this is very simple and stands against noise. For the simple implementation, the table of 60 degree interval was used. The developed sinusoidal drive for BLDC motor has been successfully applied to appliance applications.

\section{Acknowledgment}

This work was supported by the Energy Efficiency \& Resources of the Korea Institute of Energy Technology Evaluation and Planning (KETEP) grant funded by the Korea government Ministry of Knowledge Economy. (No. 20132020101850)

\section{References}

(1) Richard Valentine, Motor Control Electronics Hnadbook, McGraw-Hill Handbooks, 1998.

(2) T.J.E. Miller, and Hendershot, Design of Brushless Permanent-Magnet Motors, Magna Physics publishing and Clarendon Press, Oxford, 1994.

(3) Kwanghee Nam, AC Motor Control and Electric Vehicle Applications, CRC Press, 2010.

(4) J. Holtz, "Pulse Width Modulation. A Survey," IEEE Trans. Electron., Vol.39, No.5, pp410-420, 1992.

(5) J. Holtz, "Pulse Width Modulation for Electronic Power Conversion," Pro. IEEE, Vol.82, No.8, pp.1194-1214, 1994.

(6) D. G. Holmes and T. A. Lipo, Pulse Width Modulation for Power Converters: Principle and Practice, Hoboken, NJ: Wiley, 2003.

(7) H. W. van der Broeck, H. Ch. Skudelny and G. Stanke, "Analysis and Realization of a Pulse Width Modulator Base on Voltage Space Vectors," Proc. IEEE IAS Annual Meeting, pp.244-251, 1986.

(8) Vladimir Blasko, "A Hybrid PWM Strategy Combing Modified Space Vector and Triangle Comparison Methods," Proc. IEEE Power Electronics Specialists Conference, pp.1872-1878, 1996. 